C2008 IEEE. Personal use of this material is permitted. However, permission to reprint/republish this material for advertising or promotional purposes or for creating new collective works for resale or redistribution to servers or lists, or to reuse any copyrighted component of this work in other works must be obtained from the IEEE. 


\title{
Application of Digital Ecosystems in Health Domain
}

\author{
M. Hadzic, T.S. Dillon \\ Research Lab for Digital Health Ecosystems, Digital Ecosystems and Business Intelligence Institute, Curtin University of \\ Technology, GPO Box U1987, Perth 6845, Western Australia, Australia \\ e-mail:(m.hadzic)@ curtin.edu.au
}

\begin{abstract}
Digital EcoSystems (DES) have recently been introduced into the computer and information societies. A Digital Ecosystem is the dynamic and synergetic complex of Digital Communities consisting of interconnected, interrelated and interdependent Digital Species situated in a Digital Environment, that interact as a functional unit and are linked together through actions, information and transaction flows. Digital Ecosystems integrate various cutting-edge technologies including ontologies, agent-based and self-organizing systems, swarm intelligence, ambient intelligence, data mining etc. The synergetic effects of these methodologies results in a more efficient, effective, reliable and secure system.

The application of DES within the health domain would transform the way in which health information is created, stored, accessed, used, managed, analyzed and shared, and would bring an innovative breakthrough within health domain. In this paper, we illustrate how the DES Design Methodology can be implemented within the health domain. We focus on the key factors associated with the DES design. The design methodology framework allows better control over the design process and serves as a navigating tool during the Digital Health Ecosystems design.
\end{abstract}

Index Terms-Digital Ecosystems, Digital Ecosystems Design Methodology, Digital Health Ecosystems, Digital Health Species, Digital Health Environment

\section{INTRODUCTION}

The concept of a Digital Ecosystem (DES) has been recently adopted by the computer and information society. $\mathrm{DES}$ is analogous to the biological ecosystems in nature. It is a dynamic and complex system that is composed of a variety of interrelated digital species that interact with each other and with their digital environment [1].

The DES infrastructure is a Digital Environment (DE) which is populated by Digital Species (DS). DS are analogous to biological species and usually form communities. The majority of DS consist of hardware together with its associated software. The hardware is analogous to the body of biological species whereas the software is analogous to the life of biological species. In nature, a body without life is dead. Similarly, hardware without any application running on it is useless. DE is analogous to the biological environment. A DE is an environment in which DSs are situated and in which they live and function. DSs together with DE form a dynamic and interrelated complex DES. DES transpose mechanisms from living organisms like autonomy, viability and self-organization to arrive at novel knowledge and architectures.

We define DES as follows: Digital Ecosystem is the $d y$ - namic and synergetic complex of Digital Communities consisting of interconnected, interrelated and interdependent Digital Species situated in a Digital Environment, that interact as a functional unit and are linked together through actions, information and transaction flows.

Digital Health Ecosystem (DHES) can be specifically developed for the health domain. Various Digital Health Species (DHS) can be designed to form a collaboartive network and link different hospitals, health services, general practitioners, pharmacies, health systems, health information resources etc. producing outcomes highly beneficial for all parties involved.

The information flow can be any idea that is expressed by a formal or natural language, digitalized and transported within the DES and processed by computers or humans. In a DHES, such information may be a personalized medical record which may need to be transported within the DHES for various reasons. More complex tasks will be based on the information flows but will also involve transactions. These transactions may involve activities such as money transactions between patient and chemist when purchasing prescribed medications. A DHES should be able to autonomously perform most of these transactions with the minimal involvement of humans.

A DES transcends the traditional rigorously defined collaborative environment, such as centralized (client-server) or distributed (peer-to-peer) models into loosely coupled, domain-specific and demand driven interactive Digital Communities [1]. A DES is characterized by the beneficial activities that attract other DS to participate and benefit from it. DHES that operates successfully would attract other health institutions to join the DHES rather than compete with it. For example, a DHES can be established originally to be implemented within government health institutions and organizations. Once this DHES has proven to be successful, private health institutions and organizations may be interested to join the DHES. This will result in a strong collaborative and mutually beneficial environment.

As a new technology, DES are unknown, or vaguely represented and misunderstood, by a huge number of people. The aim of this paper is to clarify the concepts of Digital Ecosystem, Digital Species and Digital Environment and to illustrate the design of such systems within the health domain. Our ideas are inspired by the natural processes and we draw the analogy with the biological ecosystem throughout the paper. 


\section{Digital Health Ecosystems Design}

We propose a methodology for the design of DHES that consists of the following five steps:

1. Define Roles of Different DHS

2. Make the DHS Intelligent

3. Define DHS' collaborations

4. Enable, Improve and/or Construct Individual DHS

5. Protect the DHES by Implementing Security Requirements

\section{A. Define Roles of Different DHS}

A DHES functions best if it is composed of DHS with different but complementary capabilities. Medical devices, measuring apparatus, monitors, PDAs (Personal Digital Assistants), computers, etc. with different applications running on them are all different kinds of DHS that are situated within the Digital Health Environment (DHE) and all together create a DHES. These different DHS need to function as a whole; they need to work cooperatively, coordinate their actions, share the overall task, integrate their results, and so on. DHS need to be able to talk to each other without human intervention. For this reason, even though the DHS may differ from each other, they are interrelated, interconnected and interdependent since they function as a whole within the same DHES.

When considering capabilities of different DHS, it is important to:

- establish intuitive Actions, Information and Transaction (AIT) flow

- identify DHS's roles required to establish the intuitive flows

- accordingly, identify different DHS types

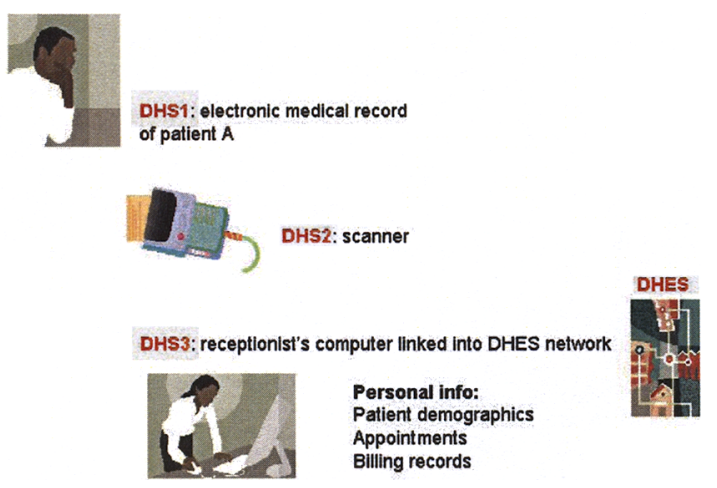

Fig. 1 Use of electronic medical records in DHES

As a DHES is composed of cooperative DHS, an intuitive action, information and transaction flow needs to be established. We follow these functional process(es) in our minds from the beginning to the end, and progressively identify different aspects of the problems. Corresponding roles that are needed to bring these process(es) to comple- tion need to be clearly defined. A diversity of roles emerges in this way. Different DS types associated with the identified roles need to be clearly specified.

We may have an example of a patient coming to a hospital and making an appointment to see a doctor (see Figures 1 and 2). We can make use of a machine-readable card (DHS1) and a scanner (DHS2) to make this information accessible and visible using a computer which is linked into the DHES network (DHS3). Depending on the access permitted to the receptionist, she would be able to read only that part of the medical record she needs to access in order to make the appointment. (The associated security and privacy issue will be discussed in Section E.) Other DHS of the DHES network inform her about the available services and timetables of the doctors so she can make an appointment relevant to the patient's need. A decision support system (DHS4) can be designed to help the receptionist in making decisions. Analogously to the biological species, we say that this DHS4 'lives' in 'symbiosis' with DHS3.

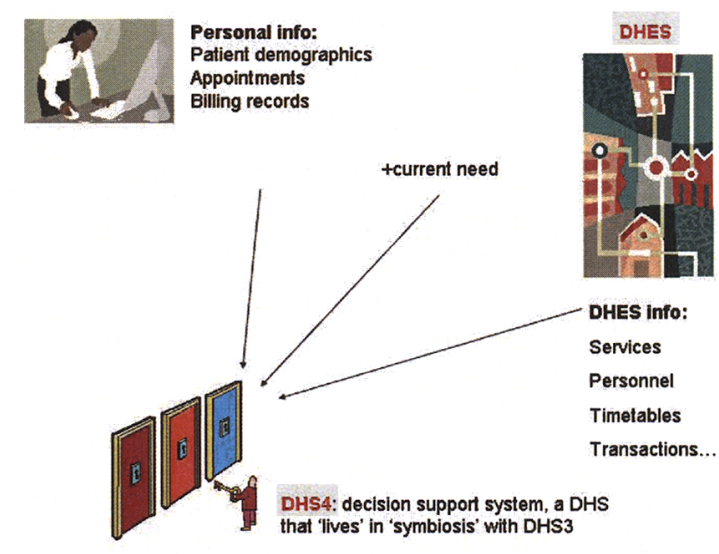

Fig. 2 Use of decision support system in DHES

\section{B. Make the DHS Intelligent}

There are different ways to provide the DHS with the intelligence. Depending on their role within DHES, each DHS will require a certain amount of intelligence. In simpler cases, a knowledge base may be used while in more complex cases, DHS need to act more intelligently and ontologies [2] may be used for this purpose. Generally, DHES should function more effectively and efficiently when based on ontologies. Ontology is used for representing the knowledge domain and may be used at different levels and for different purposes within a DHES, such as information presentation and communication between different DHS.

Machine-readable information content within a DHS can be described using an ontology. Moreover, the inherited organization of ontologies adds taxonomical characteristics to the information, and conceptual relationships in data can be easily spotted. A DHS may request information from another DHS before it performs, for example, money transactions. The use of ontologies enables the DHS to access the requested information in a more controlled and systematic 
way.

All the DHS of the DHES need to have a common understanding of the domain concepts if they are to function efficiently as a unit. Ontology provides the vocabulary needed for communication and enables cooperative DHS to communicate with each other, coordinate their actions and function as a whole unit.

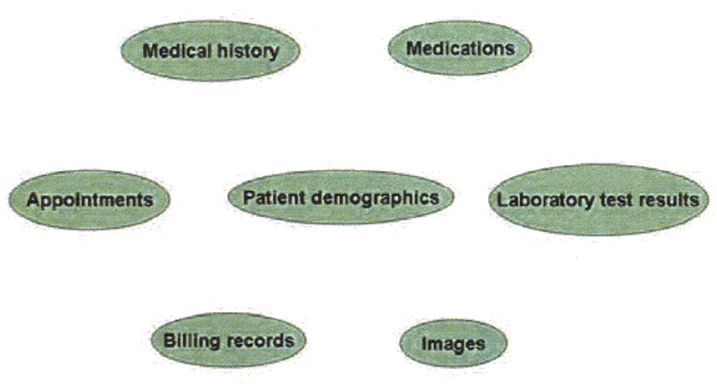

Fig. 3 Top-level ontology concepts in electronic medical record (EMR)

If the Electronic Medical Record (EMR) information is to be understood by all DHS of the DHES, standardization of data needs to take place. Ontologies can be used for this purpose [1]. Moreover, the use of ontologies adds semantics to the model and enables meaningful interpretation of the data. Generic EMR Ontology (GEMRO) can be designed to capture and represent all the EMR information and include the data about patient demographics, medical history, medications, laboratory test results, images such as radiology images or clinical photographs, appointments, billing records, etc (Figure 3). Instantiation of the GEMRO concepts results in Specific EMR Ontology (SEMRO) that act as personal medical records. DHS1 from Figure 1 is a SEMRO which is specific to this patient.

The DHS of the DHES commit to this common GEMRO. This means they obey the agreement with respect to the meaning of the concepts and relationships defined in the ontology and agree to use the ontology in a coherent and consistent manner. Because ontologies are stored as machine-readable files, DHS can read SEMROs and make this information available for the user. In Figure 1, DHS2 reads the information from DHS1 and makes it availed via DHS3 to the receptionist.

\section{Define DHS's Collaborations}

In the first phase, we described how to identify different types of DHS according to the different functions they need to perform within the DHES. In this phase, we emphasize the importance of structural organization of the DHS within a DHES. These two phases may sound similar, but the difference is that in the first phase we defined DHS' activities within a DHES, while in this phase we define the DHS' position within DHES i.e. we are concerned with inter-DS interaction.

The aim of this step is to:

- determine DHS' behaviour that will enable DHES to function in the most efficient way

- establish the structure of communication paths between the different DHS

The DHS need to be organized in such a way that the functional process within DHES can easily flow into its completion and the communication between different DHS can be established at any point. The DHES needs to function precisely and efficiently through a synergetic effort of different DHS.

DHS may have the ability of self organization [4], or their organization may be predetermined. Another possibility is that they have the ability of both self- and predetermined organization. In self organizational structure, the different DHS organize themselves to function most optimally. In predetermined organization, DHS do not have the freedom to group with each other but are communicating and collaborating as determined by their designer. This situation does not really correspond to the natural ecosystems, but this approach may be used in circumstances where greater control of DHS actions is needed. Other DHES may be designed to allow both types of DHS organization. A DHES that has the existing health institutions and organizations loosely-coupled and has a vision of other members freely joining it, needs to permit the self-organizing behaviour.

The second dimension to determine collaboration between different DHS is their structure. The different DHS can be structured in (1) chaotic, (2) orderly and (3) semiorderly way. It makes more sense that a DHES would function more efficiently if it is structured in an orderly way. These structures may take any shapes, e.g. holonic structure is associated with self-organizing, swarm intelligent behaviour. Here, the autonomous DHS group together to form hierarchies, and hierarchies of hierarchies (holons) [3].

\section{Enable, Improve and/or Construct Individual DHS}

DHS need to meet the requirements regarding their functions within the DHES. For this reason, existing DHS need to be enabled and/or improved and new DHS designed.

Some DHS are available and we do not need to redesign them e.g. PDAs, computers, monitoring devices etc. In most cases, it is needed to enable them to be used within the DHES. Some special features may need to be added to include these DHS into the dynamic network of the DHES. For example, the DHS3 from Figure 1 is a simple computer with special software enabling it to operate efficiently. We need to design the software program to access, read, understand and manage electronic medical records (SEMRO software), and another software program to schedule the appointments (Appointments software). These special features may be unique to that particular DHS, or these features may be added to all DHS of the DHES in a situation where the DHS need to perform their task collectively.

During the construction phase, it is important to:

- identify required DHS features

- design software and hardware that will support these features (Digital Organs (DO))

- assemble the DO

For all the functions that could not be covered by the ex- 
isting DHS, new DHS need to be designed. For example, if no machine-readable medical card and scanner associated with this card exist that are suitable to be used by the proposed system, we need to design these DHS. On the basis of the required functions, desired DHS features are identified and the design of corresponding Digital Organs (DO) is started. The different DO are assembled together to form DHS. In our example, the SEMRO software and Appointments software are two DO of the DHS3.

The variety of DHS system can be achieved in three different ways:

(1) Different DO used to construct different DHS are the same, but the content of the DO is different for different DHS. This approach is used when creating DHS of the same type e.g. receptionist and doctor both have a computer. As shown on Figure 4, DHS3 and DHS5 have SEMRO software in common but the SEMRO software of a receptionist differs from the SEMRO software of a doctor, as the doctor needs to have access not only to patient demographics, appointments and billing records but also to information about patients' medical history, medications, results from laboratory tests etc. In nature, this corresponds with the difference between two organism of the same species.
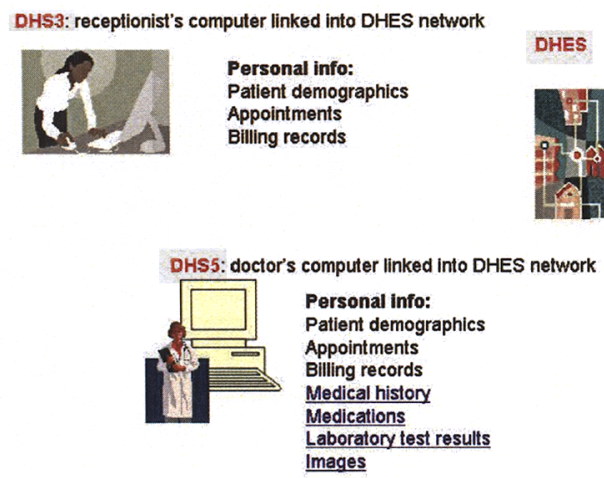

Fig. 4 Two DHS of the same type

(2) The content of the DO used to construct different agents is the same, but different DHS are constructed by a different combination of the used DO. This approach is used in situations such as designing the different DHS to function as one and linking them into interdependence where one can not exist without the other. For example, DHS4 from Figure 2 is a decision support system and needs DHS3 to be implemented on. DHS3 needs DHS4 to function intelligently. In nature, this phenomenon is commonly known as symbiosis (we are talking here only about mutualism and not about parasitism and commensalisms).

(3) The third and most common option is that different DHS differ in the combination of the DO used to construct them and in the content of these DO. The greatest diversity of DHS is created in this way. Greater variety can be achieved through a decreased number of similarities in the DO combination and their content resulting in larger differences, such as those between animals and plants in nature.
In nature, this corresponds with the variety between different species e.g. between humans, animals and plants.

In nature, no matter how complex or simple one organism is, it always has something in common with the others. This information is contained within its genetic material (DNA or RNA). Biological species are 'encoded' by unique sequences of only four different nucleotide bases: Adenine (A), Cytosine (C), Guanine (G) and Thymine (T). In the digital world, this is analogous to the sequence of atomic software encodings common to all Digital Species, namely, unique sequences of 0 and 1 .

\section{E. Protect DHES by Implementing Security Require- ments}

Security plays an important role in the development of DHES. When developing DHES, the goal is to provide as much security as possible.

Security can be supported by the existence of the properties such as [4]:

(1) Authentication: proving the identity of DHS

For example, DHS2 from Figure 1 needs to prove its identity to DHS1 in order to access the requested information.

(2) Availability: guaranteeing the accessibility and usability of information and resources to authorized DHS

Only DHS of the same type as DHS2 have access to information provided by DHS1. The information contained within DHS1 needs to be inaccessible to DS of other DES.

(3) Confidentiality: information is accessible only to authorized DHS and inaccessible to others

The amount of information that will be made accessible depends on the access allowed for a specific DHS2 type. We have seen on the example from Figure 3 that the doctor has access to information that appears invisible to the receptionist.

(4) Non repudiation: confirming the involvement of a DHS in a certain communication

DHS2 needs to confirm that it accessed information from DHS1 and not from the medical record of some other patient.

(5) Integrity: assuring that the information remains unmodified from source entity to destination entity

DHS2 must assure that the information displayed via DHS3 is identical to the information specified in DHS 1.

The abovementioned properties are critical inside the DHES as well as outside the DHES, such as during the interaction with the environment.

The aim of this design phase is to:

- identify critical security issues within the DHES

- effectively address those issues

- clearly set the boundaries

- encode this knowledge in the form that can be understood by all DHS of the DHES

- equip the DHS with this knowledge

After the identification of critical security issues and effectively addressing those issues, it is necessary to implement this knowledge within the DHES. The knowledge needs to be encoded in the format understandable by DHS. 
The DHS will use this knowledge in situations when their individual security and/or security of the DHES is in danger. They will use this knowledge to direct their actions towards protection.

For the purpose of getting more control over the security of the DHES, the designer needs to take more details into consideration. Different DHS will play different roles and, with respect to security, some will be more critical than others. As a consequence, some DHS of the system might have been assigned more security responsibilities than the others. When considering security of a DHES, it may be necessary to identify features such as:

- the role that each individual DHS plays in the security of the DHES

- actions that operate within the DHES that are most critical with respect to security

- actions that operate during the interaction of the system with the environment that are most critical with respect to security

- factors outside the DHES that are most critical with respect to security

- the part of the DHES that is most susceptible to attack from the outside

It is very important to provide each DHS with the basics knowledge that will protect it from malicious actions. Each DHS needs to be equipped with this knowledge as the enemy will always study the system and try to attack the weakest spots. Other important factors here are senses of belonging and DHES community. If some DHS are under attack, other members of the DHES need to make their best efforts to protect those DHS and the whole DHES.

\section{OVERALL DISCUSSION}

The design of Digital Health Ecosystem can be followed through the five phases represented in Figure 5. When the preliminary DHES gets implemented, some additional requirements arise so that the corresponding design phases need to be repeated until the designer is satisfied with the outcomes.

In the first phase, Define Roles of Different DHS, intuitive Action, Information and Transaction (AIT) flow is determined, required DHS roles identified and corresponding DHS's types proposed. The second design phase, Make the DHS Intelligent, deals with the issue of DHS' intelligence. Because ontologies are the most expressive knowledge models and enable the system to function the most efficiently, the ontologies were discussed predominantly. Analogous ways of thinking are applicable for other approaches. In the third phase, Define DHS' Collaboration, DS' behaviour was defined (which can be self-organization, predetermined organization and self- and predetermined organization) as well as their structure (which can be chaotic, semi-orderly and orderly). In the fourth phase, Enable, Improve and Construct Individual DHS, special features are added to the existing DHS to enable them to function efficiently within the DHES, and new DHS are designed. The design of new DHS includes identification of the desired features, designing of DO to mirror these features and assembling of the DO into the DHS. Variety of DHS is a result of variety of the DO and their contents. In the fifth phase, Protect the DHES by Implementing Security Requirements, the security issues of authentication, availability, confidentiality, non repudiation and integrity are carefully addressed and the boundaries clearly set. This knowledge is encoded in the common language and given to the DHS.

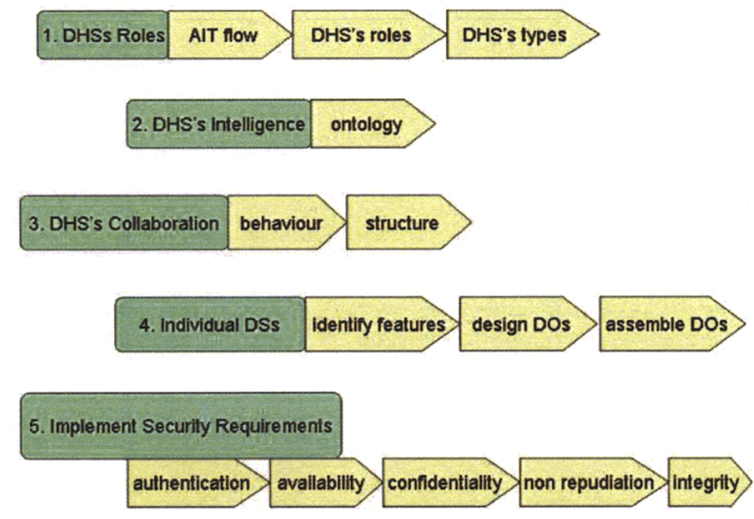

Fig. 5 Digital Ecosystem Design Methodology

\section{CONCLUSION AND FUTURE WORK}

This paper can bring in a better and clearer understanding of a DHES and its design, and design of DES in general. The stepwise insight provided into the design process increases the control over the design process. The role of each step in the complete design process was discussed and an analogy with biological ecosystems was provided. An example of Digital Health Ecosystem and specifically the handling of electronic medical records within this system was used to illustrate the implementation of this methodology framework within the health domain. We only gave a brief overview and the major steps associated with the DHES design. This methodology is to be improved and refined as more experience is gained with the design of such systems.

\section{REFERENCES}

[1] M. Hadzic, T. Dillon, E. Chang, 'Use of Digital Ecosystem and Ontology Technology for Standardization of Medical Records', Proceedings of the Inaugural IEEE International Conference on Digital Ecosystems and Technologies (DEST 2007).

[2] T. Gruber, "Towards principles for the design of ontologies used for knowledge sharing", International Journal of Human and Computer Studies 1995, vol. 43, no. 5-6, pp. 907-928.

[3] M. Ulieru, "Internet-Enabled Soft Computing Holarchies for e-Health Applications", New Directions in Enhancing the Power of the Internet, 2003, pp. 131-166

[4] H. Mouratidis, P. Giorgini, G.A. Manson, "Modelling Secure Multiagent Systems", in the Proceedings of the Second International Joint Conference on Autonomous Agents and Multiagent Systems (AAMAS 2003), pp. 859-866. 